\section{Post-pneumonectomy bronchopleural fistula: complex surgical repair for a high mortality case}

\author{
Devender Singh,' Shegu Gilbert, ${ }^{1}$ \\ Manohar Arumugam, ${ }^{2}$ \\ Paulvannan Subramanian, ${ }^{3}$ \\ Sivakumar Mk, ${ }^{4}$ Srinivasa Ramachandra ${ }^{4}$ \\ 'Department of Cardiothoracic Surgery, \\ Kovai Medical Center and Hospital, \\ Coimbatore; ${ }^{2}$ Department of Plastic \\ Surgery, Kovai Medical Center and \\ Hospital, Coimbatore; ${ }^{3}$ Department of \\ General Surgery, Kovai Medical Center \\ and Hospital, Coimbatore; ${ }^{4}$ Department \\ of Cardiac Anesthesia, Kovai Medical
}

Center and Hospital, Coimbatore, India

\section{Abstract}

Post-pneumonectomy empyema and bronchopleural fistula are dreaded complications of thoracic surgery. The associated mortality and morbidity is very high. When treatment is delayed secondary complications are more likely and survival is adversely affected. These conditions require early diagnosis and a wellplanned multi-disciplinary team approach for effective management. We present a patient with post-pneumonectomy empyema and bronchopleural fistula following pneumonectomy for post tuberculosis destroyed lung. He underwent debridement of the empyema cavity, double layer closure of the bronchial stump and reinforcement with omental flap and pectoralis major flap was used to obliterate the space. Debridement and pedicle flaps are safe and effective in the definitive management of postpneumonectomy empyema and bronchopleural fistulae.

\section{Introduction}

Post-pneumonectomy empyema (PNE) is a severe complication of thoracic surgery, and most cases are associated with bronchopleural fistula (BPF). ${ }^{1}$ The incidence of PNE is approximately $5 \%$, and the occurrence of BPF is $>80 \%$ in these cases. ${ }^{2,3}$ The mortality rate following PNE with a BPF has been reported to be 11 to $13 \%{ }^{3}$ Persistent BPFs often are associated with multiple operations and prolonged hospitalization. Occasionally, small uncomplicated BPF may heal spontaneously, and in 20\% of patients BPF will close with drainage only. However, the remaining $80 \%$ of patients whose
BPF persist will require additional operative procedures.

\section{Case Report}

A 32-year-old gentleman presented to us with history of chronic cough with expectoration since eight months. He gave history of difficulty on lying down on the left side. He had undergone right pneumonectomy for post tuberculosis destroyed right lung. Two months after surgery, he started having cough with expectoration especially while lying on left side. There was no history of fever. Patient was cachectic. Bronchoscopy showed right lower lobe stump dehiscence. Culture of bronchial secretions showed growth of Klebsiella pneumoniae. Chest $\mathrm{X}$ ray showed minimal right pleural collection. A computerized tomography (CT) of chest demonstrated a BPF from the right lower bronchial stump site and empyema (Figure 1A). He was treated with culture specific antibiotics for two weeks prior to the planned surgery.

The patient was planned for the complex surgical repair with multi-specialty team consisting of thoracic, plastic and gastrointestinal surgeons. The entire surgical plan was documented and the outcome was discussed with the patient and family members and a written consent was taken.

The surgery was done with general anesthesia and double lumen endotracheal tube for protecting the normal left lung. The surgery involved three well demarcated procedures. First, initial debridement of the pleural cavity and washing with normal saline was done. This was followed by excision and debridement of the bronchial stump. The stump site was closed in a double layer fashion with interrupted polypropylene (Centenial sutures, India) sutures and reinforced with a Dacron patch.

The second stage performed by gastrointestinal surgeon involved laparoscopic mobilization of omentum \& its placement in the right pleural space across the diaphragm. The omentum was wrapped around the bronchial stump (Figure 1B).

The third stage, performed by plastic surgeon, consisted of harvesting pectoralis major (PM) flap with vascular pedicle and rotating and placing it in right pleural cavity through an opening made by excising a part of the third rib (Figure 1C). The PM flap was used to fill up the residual pleural cavity. Thoracotomy was closed in a routine fashion in three layers with one intercostal drain and one Romovac suction drain in the bed of the PM muscle.

The patient was extubated after $4 \mathrm{~h}$. Postoperative chest X-ray showed resolution of empyema (Figure 1D). The intercostal drain was removed on the third post-operative day
Correspondence: Shegu Gilbert, Department of Cardiothoracic Surgery, Kovai Medical Center and Hospital, Avinashi Road, Coimbatore, Tamil Nadu, India.

Tel: +91.9840242143

E-mail: shegugilbert@yahoo.co.in

Key words: Empyema; Pneumonectomy; Tuberculosis; Fistula.

Contributions: DS, SG, MA, PS,S SMk, SR, study conception and design, acquisition of data, analysis and interpretation of data, drafting of manuscript, critical revision.

Conflict of interest: the authors declare no potential conflict of interest.

Received for publication: 22 December 2016 Revision received: 30 May 2016.

Accepted for publication: 29 June 2016.

This work is licensed under a Creative Commons Attribution NonCommercial 4.0 License (CC BYNC 4.0).

(C) Copyright D. Singh et al., 2016

Licensee PAGEPress, Italy

Chest Disease Reports 2016; 4:5696

doi:10.4081/cdr.2016.5696

and the suction drain was removed on the fifth post-operative day was discharged on postop day 10. Intravenous antibiotics were given for 7 days. Follow-up is limited to 6 months. He did not show any evidence of recurrent BPF. Post operative chest radiograph did not show any signs of BPF and the patient was asymptomatic.

\section{Discussion}

Various systemic factors and therapeutic interventions often contribute to the risk of PNE and BPF, including advanced age in men ( $>70$ years), preoperative radiation, malnutrition, and prolonged steroid therapy. ${ }^{3}$ In addition, technical factors such as prior lung resection, infection at a long bronchial stump site and residual sepsis in the pleural space may further contribute to the development of this complication. Other high risk conditions leading to BPF include improper surgical technique, devascularization of the stump due to extensive cicumferential dissection and residual tumour on the stump. Non surgical conditions leading to BPF include uncontrolled diabetes mellitus, hypoalbunemia, cirrhosis and steroid administration and post-pneumonectomy ventilation for more than $24 \mathrm{~h}$. Incidence of BPF in tuberculosis patients undergoing lung surgeries is between 0 to $16 \%$. Investigations for BPF include plain chest radiograph which 

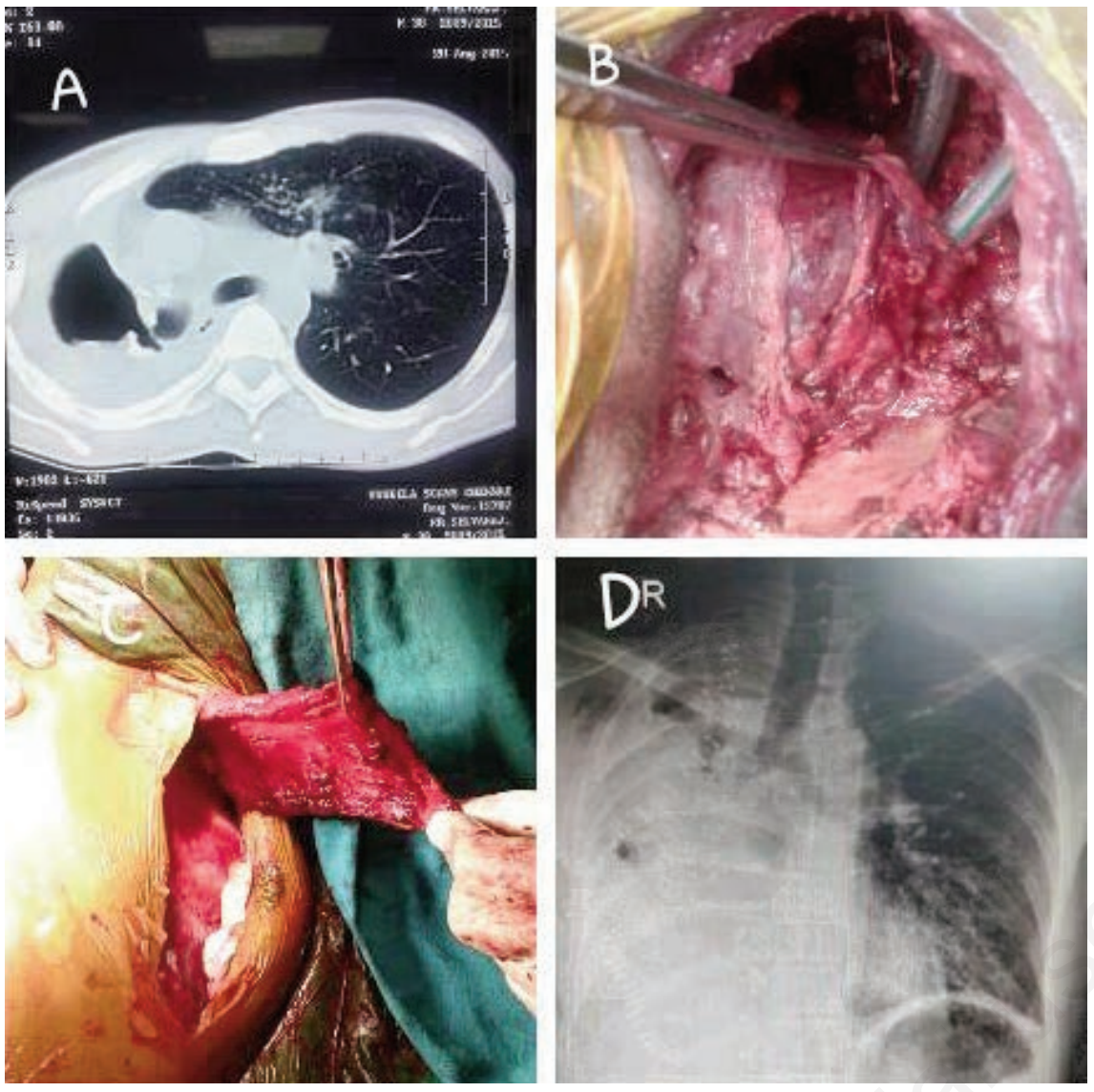

Figure 1. A) Computerized tomography of chest showing right lower lobe bronchopleural fistula and empyema; B) omentoplasty - omentum mobilized into the pleural cavity; C) right pectoralis major flap for thorocomyoplasty; D) post-operative chest $\mathrm{X}$-ray showing resolution of the empyema.

shows enlargement of a previously small space or a newly formed air fluid level may be noted. Other radiological signs include lowering or sudden disappearance of a pleural effusion or a mediastinal shift towards the contralateral side. The diagnosis is usually made by fiberoptic bronchoscopy (FOB) or by observing persistent air leak through the chest tube. CT of the chest and 3D reconstruction from the volume acquistion using spiral tecnique can identify the BPF in some cases. FOB and selective bronchography can also be done. Capnography can be used. Isotope studies like ${ }^{133}$ Xenon, ${ }^{81}$ Krypton and ${ }^{99}$ Technetium can be used. Visualization of equilibration of radioactive gas tracer into the pleural space in occult bpf can help in the diagnosis. Previous interventions like closed tube thorocostomy drainage and negative pressure therapy is not very effective. Negative pressure increases the flow through the fistula. Ambulatory drainage is also not very effective in the management of BPF. The treatment strategy for empyema with BPF, consists of closure of the fistula and obliteration of the empyema cavity. Radical surgery can be accomplished by two procedures: single stage closure, or obliteration after open window thoracotomy (OWT). ${ }^{4}$ For obliteration of the empyema cavity following OWT, the materials used are usually omentum and local chest wall muscles overlying the cavity, abdominal wall muscles, or other vascularized muscles. In our patient we have done double layer closure of the bronchial stump and omentum was wrapped around to promote vascularization and healing. The PM flap was used to obliterate the cavity and promote healing. Overall, PNE and BPF are best prevented by minimization of perioperative sepsis, careful closure of the bronchial stump, and the use of vascularized flaps to reinforce the bronchial stump. ${ }^{5}$ Patients with known preoperative infections should have appropriate culture tests and antibiotic therapy prior to operative procedures. The integrity of the bronchial stump closure in the post-operative period can be confirmed clinically by the absence of breath sounds in the operated side of the chest, absence of air leak in the intercostal drain, by plain chest radiograph and bronchoscopy. Non-surgical procedures for closure of BPF include the use of cyanoacrylate compounds, fibrin or tissue glue and gel foam. ${ }^{6}$ Bronchial blockades in the form of intrabronchial valves and vascular embolization coils can be used. Placement of endobronchial stents can also be done. Bronchoscopic submucosal injections with ethanol and other compounds are also reported.

\section{Conclusions}

We present a successful treatment of PNE with BPF with a well-coordinated team approach. Our patient is an example of delayed dehiscence of bronchial stump. Our approach can be used in these high mortality risk patients for a successful outcome.

\section{References}

1. Pralay S, Twinkle C, Rakesh S, Arunabh T. Diagnosis and management bronchopleural fistula. Indian J Chest All Sci 2010;52: 97-104.

2. Gharagozloo F, Tracniotis G, Wolfe A, et al. Pleural space irrigation and modified Clagett procedure for the treatment of early postpneumonectomy empyema. Thorac Cardiov Surg 1998;16:943-8.

3. Hochberg J, Ardenghy M, Yuen J, et al. Utilization of muscle flaps in the treatment of bronchopleura fistulas. Ann Plast Surg 1999;43:484-3.

4. Masaya 0, Hiroyasu Y, Shintaro T, ChenLong H. Non-surgical closure of post-pneumonectomy empyema with bronchopleural fistula after open window thoracotomy using basic fibroblast growth factor. Interact Cardio Vasc Thorac Surg 2009;9: 916-8.

5. Eugeniusz J. Postpneumonectomy empyema. Eur J Cardio-Thorac 1998;14:123-6.

6. Lois M, Noppen M. Bronchopleural fistulas: an overview of the problem with special focus on endoscopic management. Chest 2005;128:3955-65. 